\title{
Characterizing dissolved organic matter across a riparian soil-water interface: Preliminary insights from a molecular level perspective
}

Kai Wang ${ }^{1,2}$, Yu Pang ${ }^{1}$, Yunyun $\mathrm{Li}^{4}$, Chen $\mathrm{He}^{4}$, Quan $\mathrm{Shi}^{4}$, Yuntao Wang ${ }^{3}$, Ding $\mathrm{He}^{1,3^{*}}$

${ }^{1}$ Key Laboratory of Geoscience Big Data and Deep Resource of Zhejiang Province, School of Earth Sciences, Zhejiang University, Hangzhou, China

${ }^{2}$ State Key Laboratory of Hydro-science and Engineering, Department of Hydraulic Engineering, Tsinghua University, Beijing 100084, China

${ }^{3}$ State Key Laboratory of Satellite Ocean Environment Dynamics, Second Institute of Oceanography, Ministry of Natural Resources, Hangzhou, China

${ }^{4}$ State Key Laboratory of Heavy Oil Processing, China University of Petroleum, Changping District, Beijing, China

Corresponding atdinghe@zju.edu.cn (DH)

\section{Contents of the Supplementary file}

Supplementary methods

Supplementary figures (S1 to S9)

Supplementary tables (Table S1 to S4)

\section{Supplementary methods}

1. The description of parameters used in optical and molecular analysis of DOM.

\begin{tabular}{ll}
\hline Parameters & Description \\
\hline Specific ultraviolet absorbance at & Absorbance per unit carbon. Typically, a higher \\
$254 \mathrm{~nm}\left[\mathrm{SUVA}_{254}\left(\mathrm{~L} \mathrm{mg-C}-1 \mathrm{~m}^{-1}\right)\right]$ & number is associated with greater aromatic content. \\
Fluorescence index (FI) & Shown to identify the relative contribution of \\
& terrestrial and microbial sources to the DOM pool. \\
Humification index (HIX) & An indicator of humic substance content or extent of \\
\hline
\end{tabular}


humification. Higher values indicate an increasing degree of Humification.

Biological index (BIX)

Aromaticity index $\left(\mathrm{AI}_{\mathrm{mod}}\right)$

Double bond equivalent (DBE)

Molecular lability index $\left(\mathrm{MLB}_{\mathrm{L}}\right)$
An indicator of autotrophic productivity. High values correspond to recently produced DOM of autochthonous origin.

An indicator of aromaticity. High values indicate high aromaticity of DOM.

An indicator related with unsaturation of molecule, High values corresponding to high unsaturation. An indicator to evaluate the lability of DOM. High values corresponding to high lability.

\section{The detailed information of the measurement of UV-visible spectral and 3D-EEMs}

UV-Visible spectral measurements were carried out on an UV-Visible Spectrophotometer using a Milli-Q water as control group in a $1 \mathrm{~cm}$ path length quartz cuvette over 230 to $650 \mathrm{~nm}$ range of scanning with $1 \mathrm{~nm}$ intervals. 3D-EEM was measured using an Aqualog (Horiba, Japan) absorption-fluorescence spectrometer with a $1 \mathrm{~cm}$ path length quartz cuvette, at emission wavelengths ranging from 210 to $620 \mathrm{~nm}$ on $3.3-\mathrm{nm}$ increments and at excitation wavelengths ranging between 240 and $600 \mathrm{~nm}$ on 3-nm increments respectively. Scan integration time was $1 \mathrm{~s}$.

\section{Incubation experiments and the definition of bio-resistant, photo-resistant, and bio- and photo-resistant molecular pools:}

Considering the river fluidity, the incubation experiments were conducted on downstream river water sample to capture the most molecules and avoid uncertainty of molecular diversity between different sample sites.

Bio-degradation experiment: duplicated in-situ water samples $(0.5 \mathrm{~L})$ from the downstream site were placed in 1L Nalgene sampling bottles with sufficient head space, tightly wrapped in aluminum foil, and placed in a dark place at $24^{\circ} \mathrm{C}$ for 30 days of continuous culture.

Photo-degradation experiment: duplicated in-situ water samples $(0.5 \mathrm{~L})$ from the downstream site (Fig. 1) were filtered through a $0.22 \mu \mathrm{m}$ filter (polycarbonate, Millipore) was placed in $1 \mathrm{~L}$ quartz bottles, and the bottle mouth was tightly sealed with a cap provided with the quartz bottle, and irradiated for 30 days under natural light.

Duplicate dark control samples $(0.22 \mu \mathrm{m}$ filtered $)$ were placed alongside the incubation samples. 
Both incubated and control samples were filtered according to the natural sample treatment method after the end of the cycle to obtain the cultured DOM samples, and then the FT-ICR MS test was performed to obtain the results.

Molecular formulas were classified based upon their stability as:

1) Bio-resistant: molecules present in both the initial and duplicates of the bio-incubated samples;

2) Photo-resistant: molecules present in both the initial and duplicates of the irradiated samples, and;

3) Bio- and photo-resistant: molecules that survive both biodegradation and irradiation, and are present in the initial, duplicates of the bio-incubated samples, and duplicates of the photoincubated samples.

In defining these above groupings, it should be noted that electrospray ionization is selective, and that this selectivity results in one peak's intensity changing relative to decreases or increases in other peak intensities. Therefore, a compound that appeared to be resistant during the incubation may also have been partially produced or degraded. That's why we used duplicated control and incubations (both bio- and photo-incubations) to further constrain the above groupings.

We further compared the directly and indirectly released DOM pools with the bio-resistant, photo-resistant, and bio- and photo-resistant pools to identify the lability of molecules within the directly and indirectly released DOM pools. 


\section{Supplementary figures and tables}
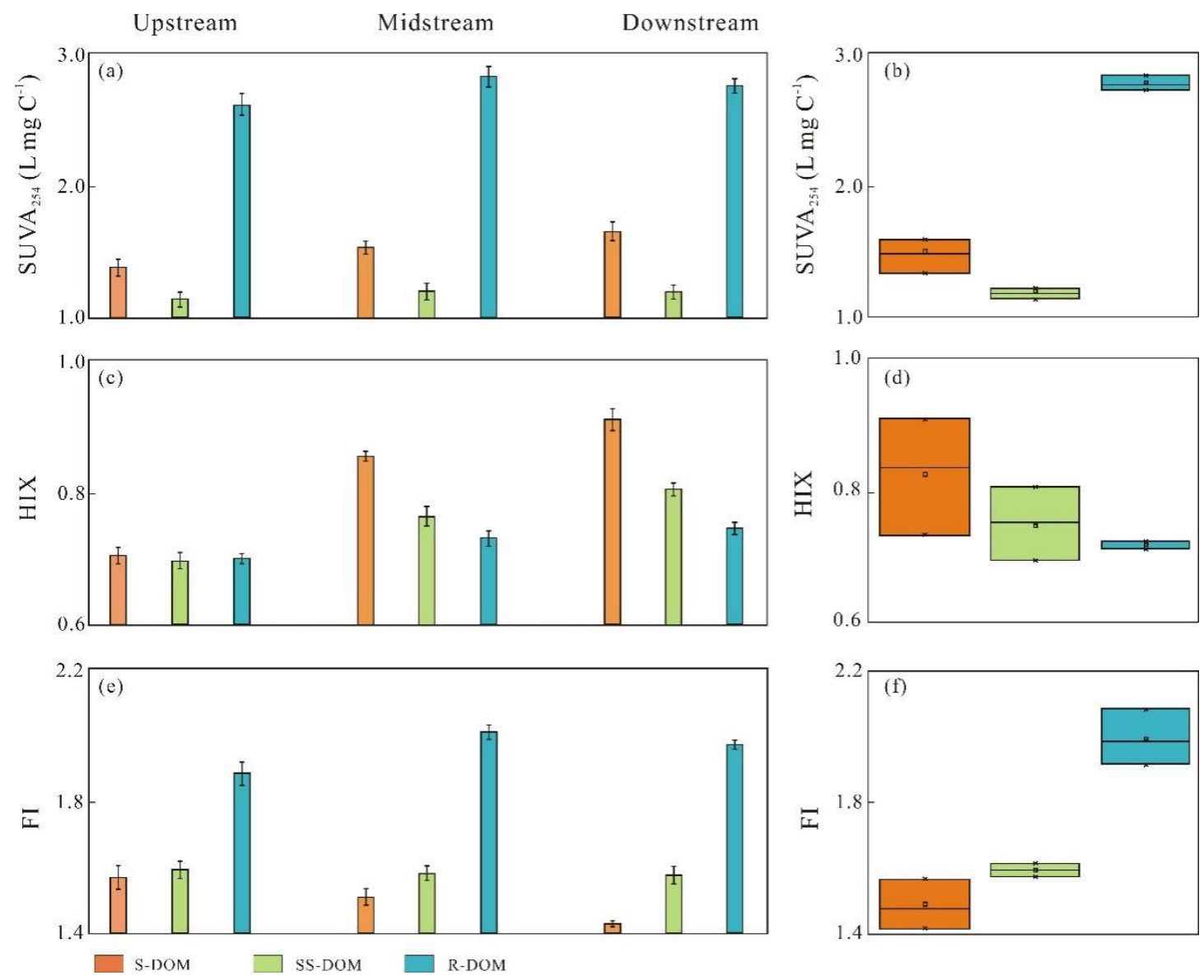

Fig. S1. Variation in optical parameters of DOM among S-DOM, SS-DOM, and R-DOM: (a) bar chart of $\mathrm{SUVA}_{254}$, (b) Box whisker plot of $\mathrm{SUVA}_{254}$, (c) bar chart of HIX, (d) box whisker plot of HIX, (e) bar chart of FI, (f) box whisker plot of FI. Error bars in (a), (c) and (e) were derived from the duplicated measuring ( $\mathrm{n}=3$ in S-DOM, SS-DOM, and R-DOM, respectively). In (b), (d), and (f) for S-DOM, SS-DOM, and R-DOM, the box symbol denotes the mean value of the dataset, with the horizontal lines in the box denoting the 25 th, 50 th, and 75 th percentile values ( $\mathrm{n}=9$ in S-DOM, SSDOM, and R-DOM, respectively). 


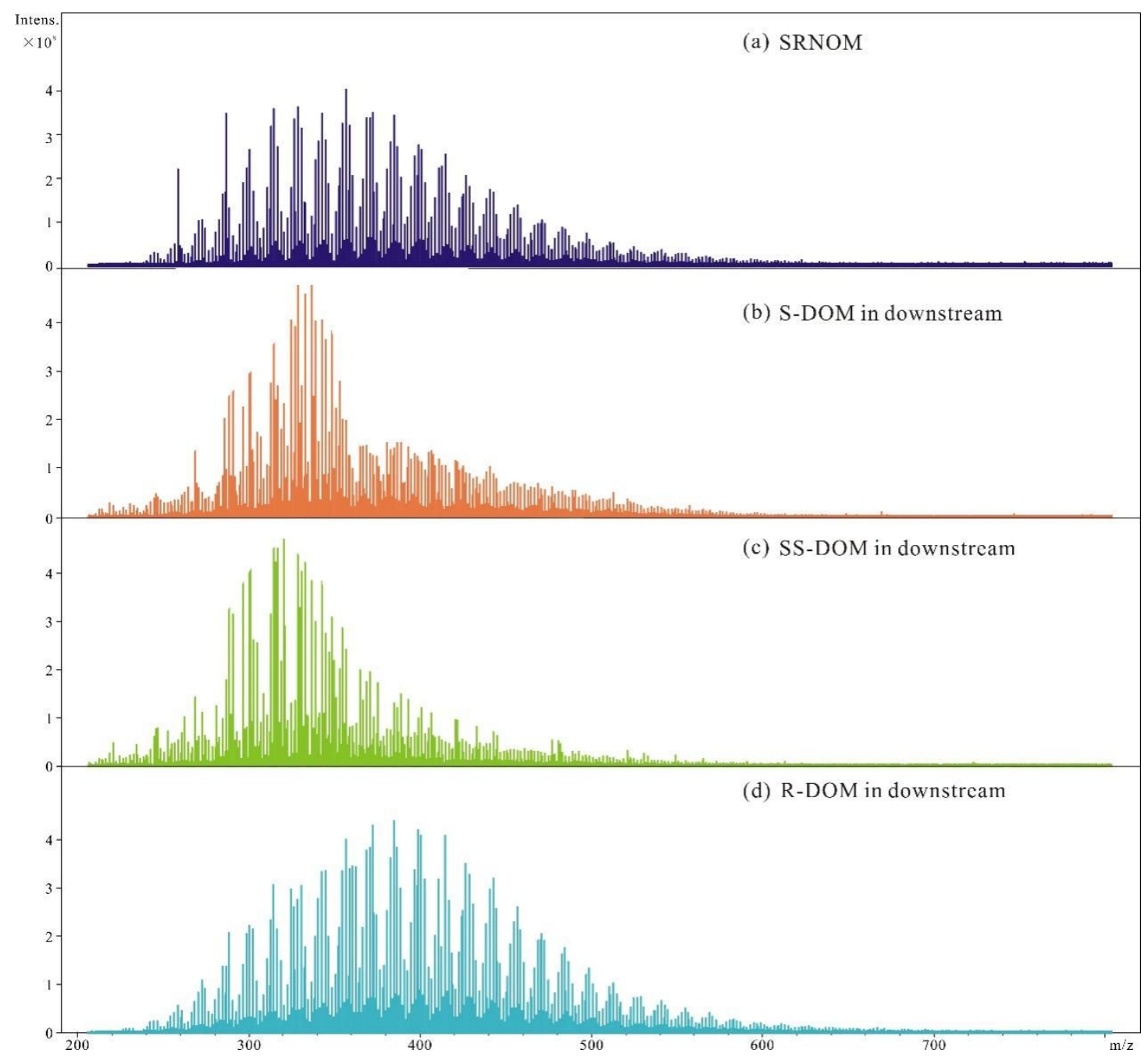

Fig. S2. Raw mass spectrum revealed by FT-ICR MS: (a) SRNOM:: standard sample, (b) S-DOM, (c) SS-DOM, (d) R-DOM in downstream. 

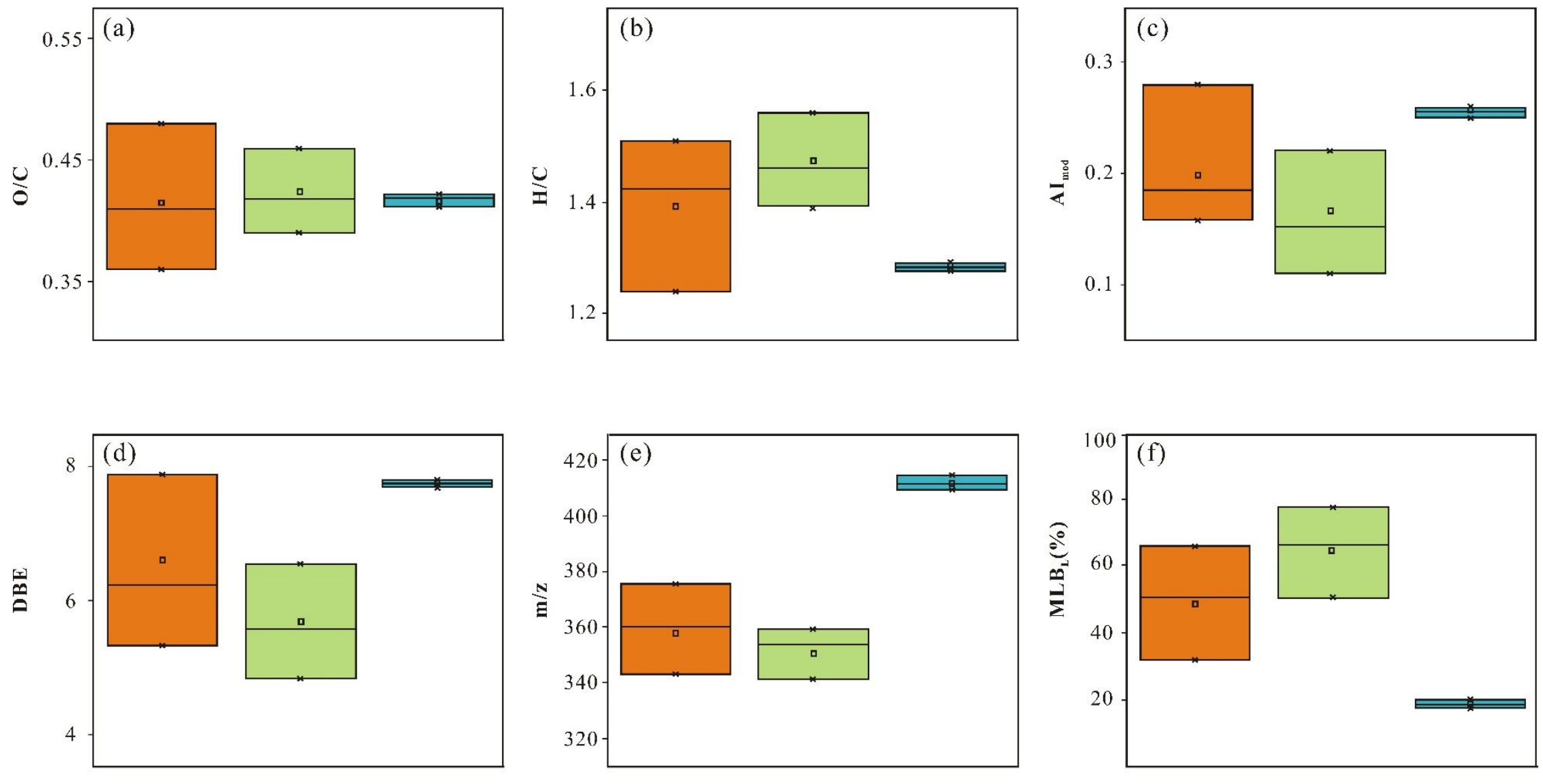

Fig. S3 Box whisker plot of optical and molecular parameters of S-DOM, SS-DOM, and R-DOM: (a) O/C, (b) H/C, (c) AI $\mathrm{I}_{\mathrm{mod}}$, (d) DBE, (f) m/z, (f) MLB $\mathrm{L}_{\mathrm{L}}$. In (a)-(f), for S-DOM, SS-DOM, and R-DOM, the box symbol denotes the mean value of the dataset, with the horizontal lines in the box denoting the 25 th, 50 th, and 75 th percentile values. 

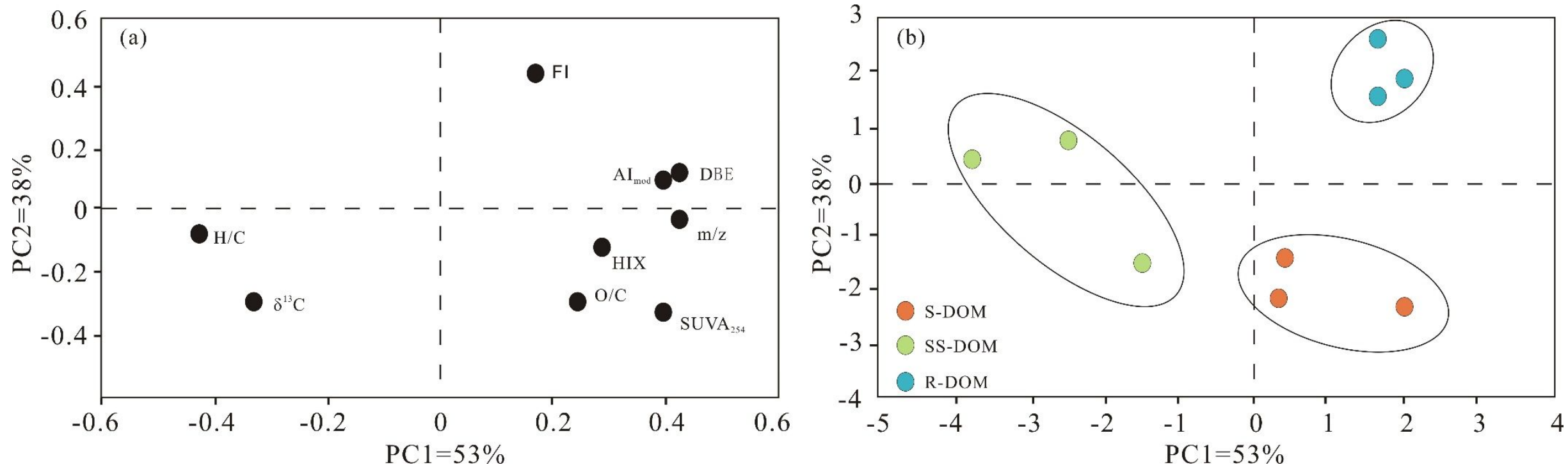

Fig. S4. Principal component analysis (PCA) of S-DOM, SS-DOM, and R-DOM based on bulk, optical, and molecular parameters: (a) loading of different parameters $\left(\delta^{13} \mathrm{C}, \mathrm{H} / \mathrm{C}, \mathrm{O} / \mathrm{C}, \mathrm{SUVA}_{254}, \mathrm{HIX}, \mathrm{FI}, \mathrm{AI}_{\bmod }, \mathrm{m} / \mathrm{z}\right.$, and DBE), (b) corresponding scores of the samples (S-DOM, SS-DOM, and R-DOM) 


\section{CHO}
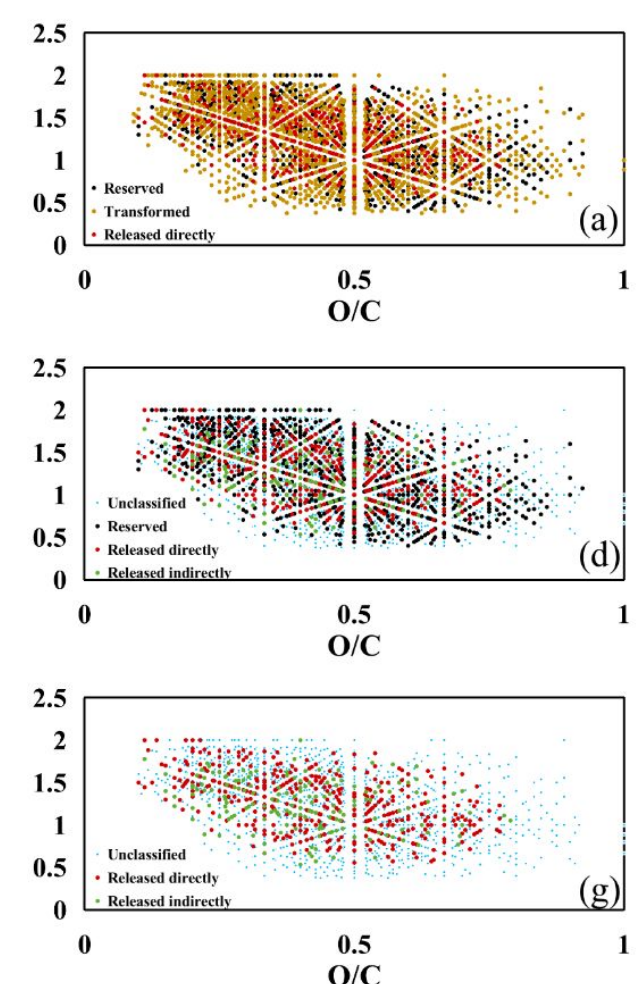

CHON
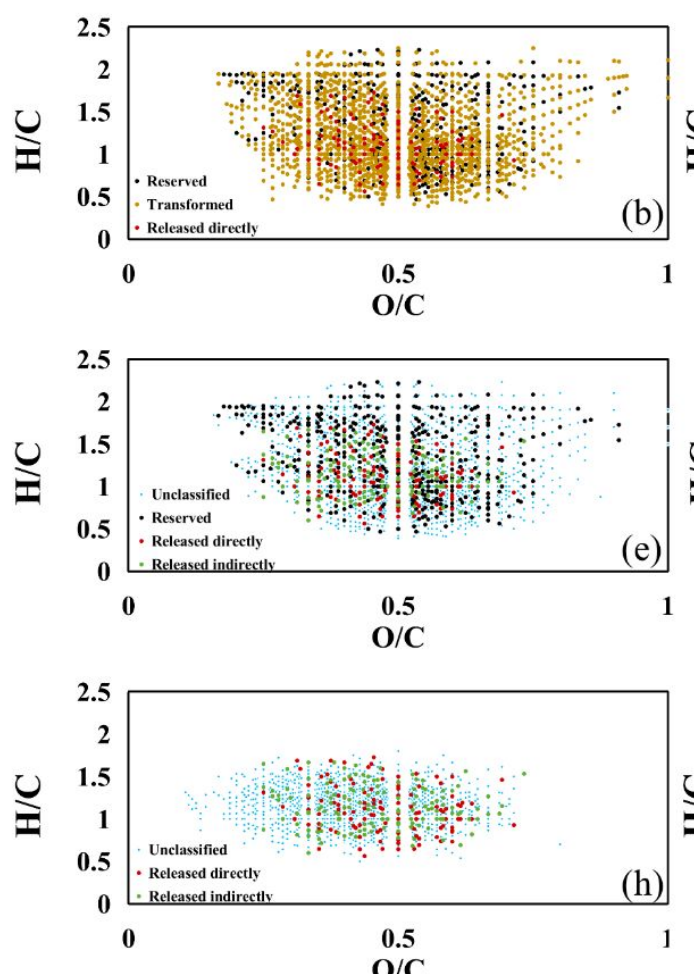

\section{CHOS}
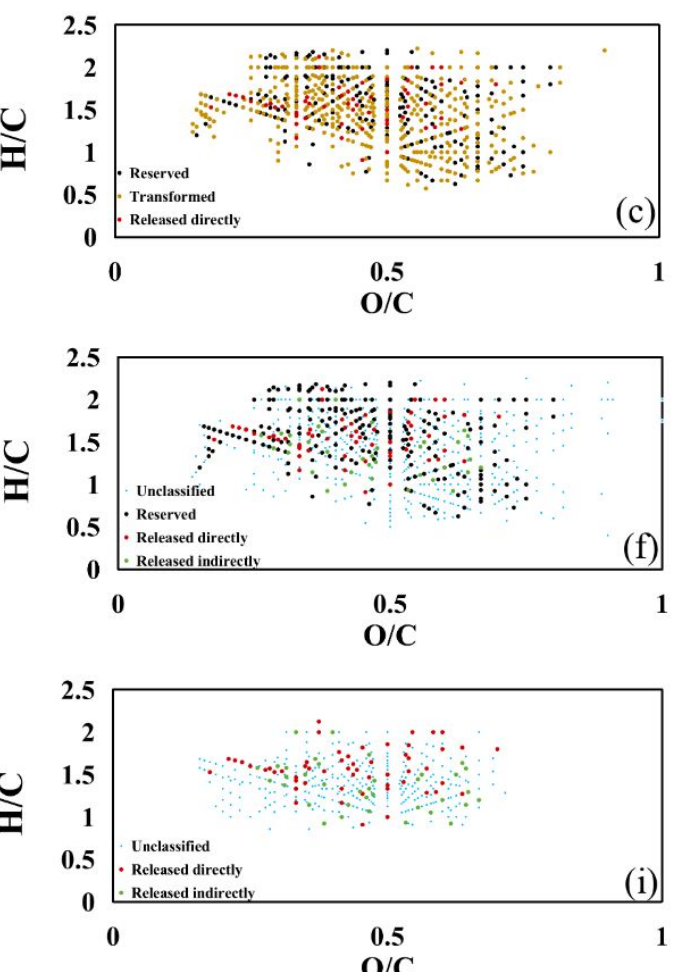

SS-DOM

S-DOM

R-DOM

(2)

Fig. S5. Van Krevelen diagrams of DOM from CHO (a), CHON (b), CHOS (c) in S-DOM with molecular pools (Reserved, Transformed, and Released directly) identified; CHO (d), CHON (e), CHOS (f) in SS-DOM with molecular pools (Unclassified, Reserved, Released directly, and Released indirectly) identified; CHO (g), CHON (h), CHOS (i) in R-DOM with molecular pools (Unclassified, Released directly, and Released indirectly) identified. 

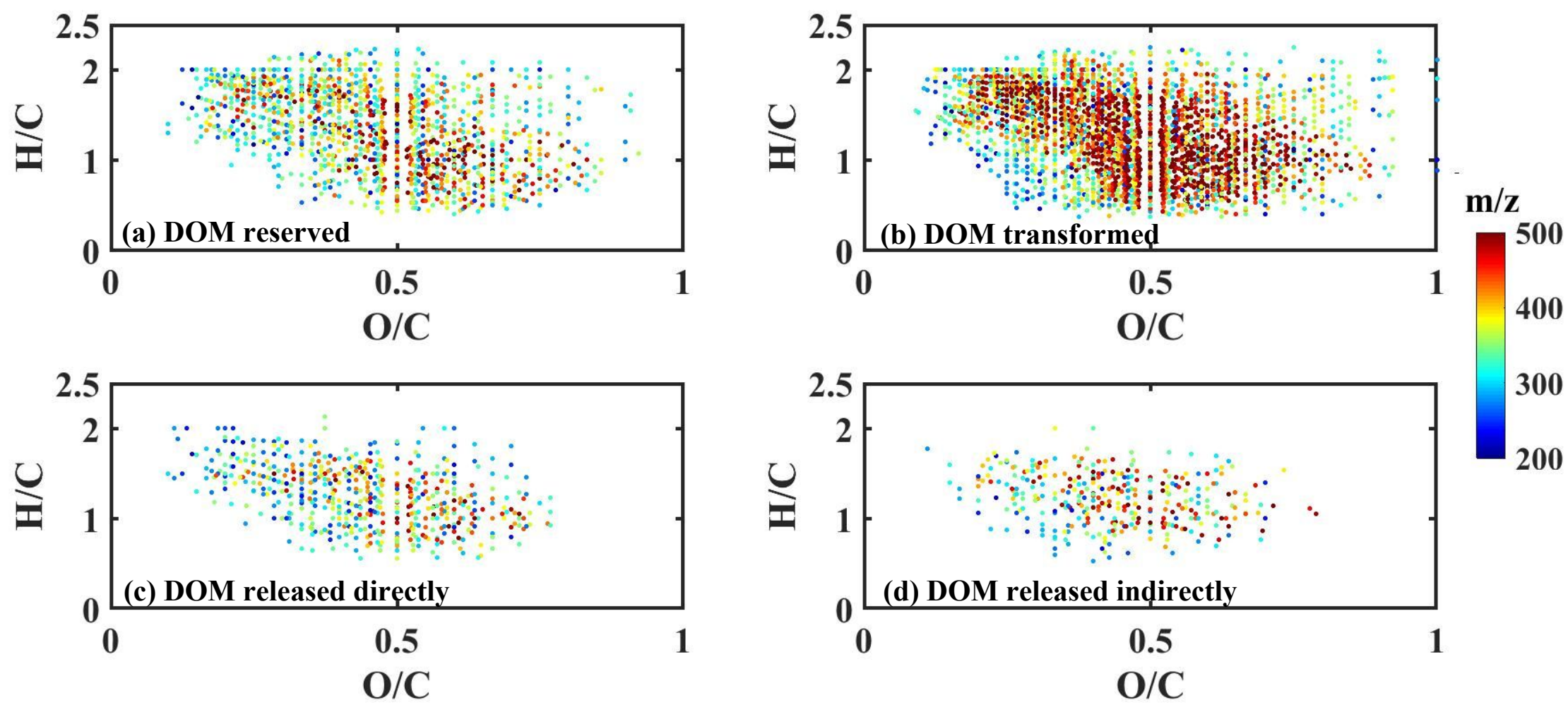

Fig. S6. Van Krevelen diagrams of different DOM pools with m/z: (a) DOM reserved, (b) DOM transformed, (c) DOM released directly, (d) DOM released indirectly. 

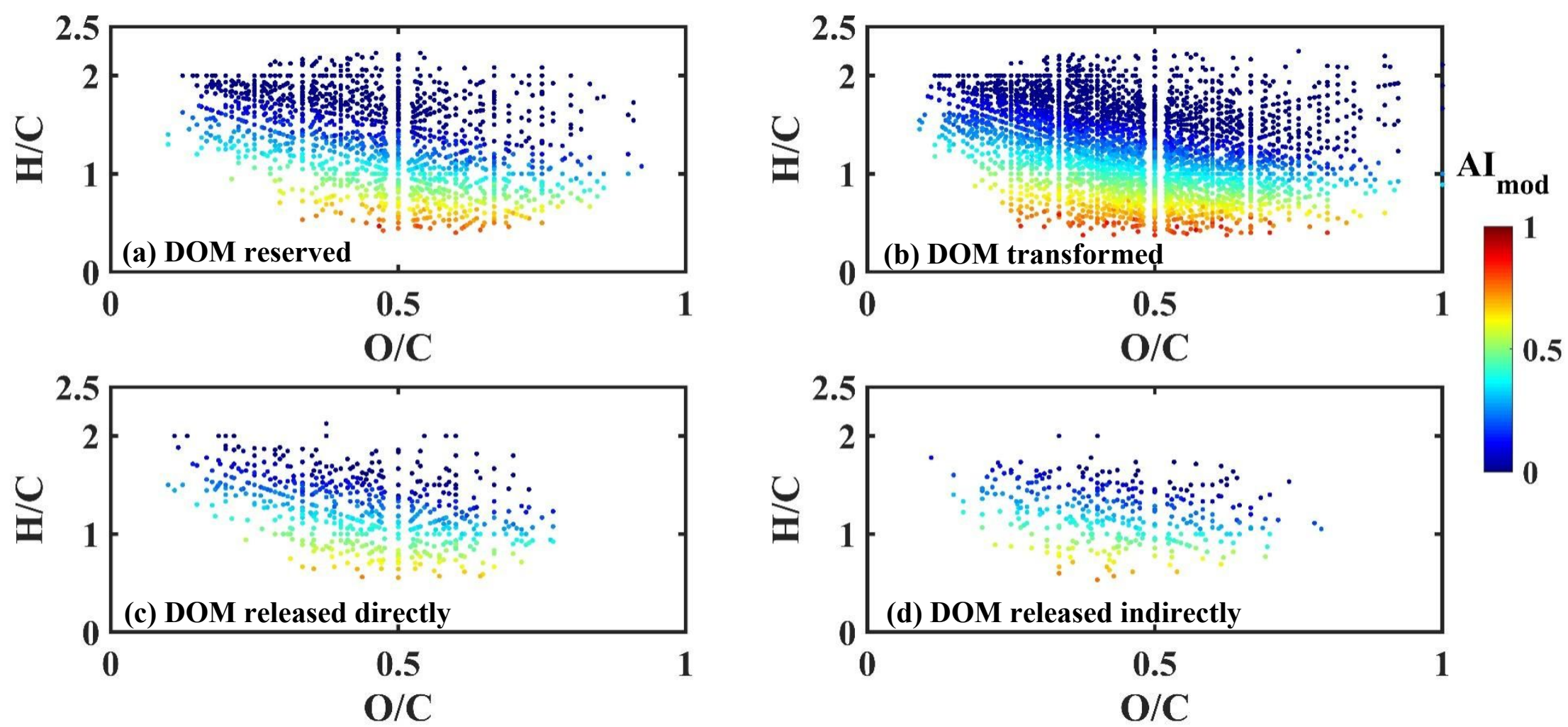

Fig. S7. Van Krevelen diagrams of different DOM pools with $\mathrm{AI}_{\text {mod }}$ : (a) DOM reserved, (b) DOM transformed, (c) DOM released directly, (d) DOM released indirectly. 

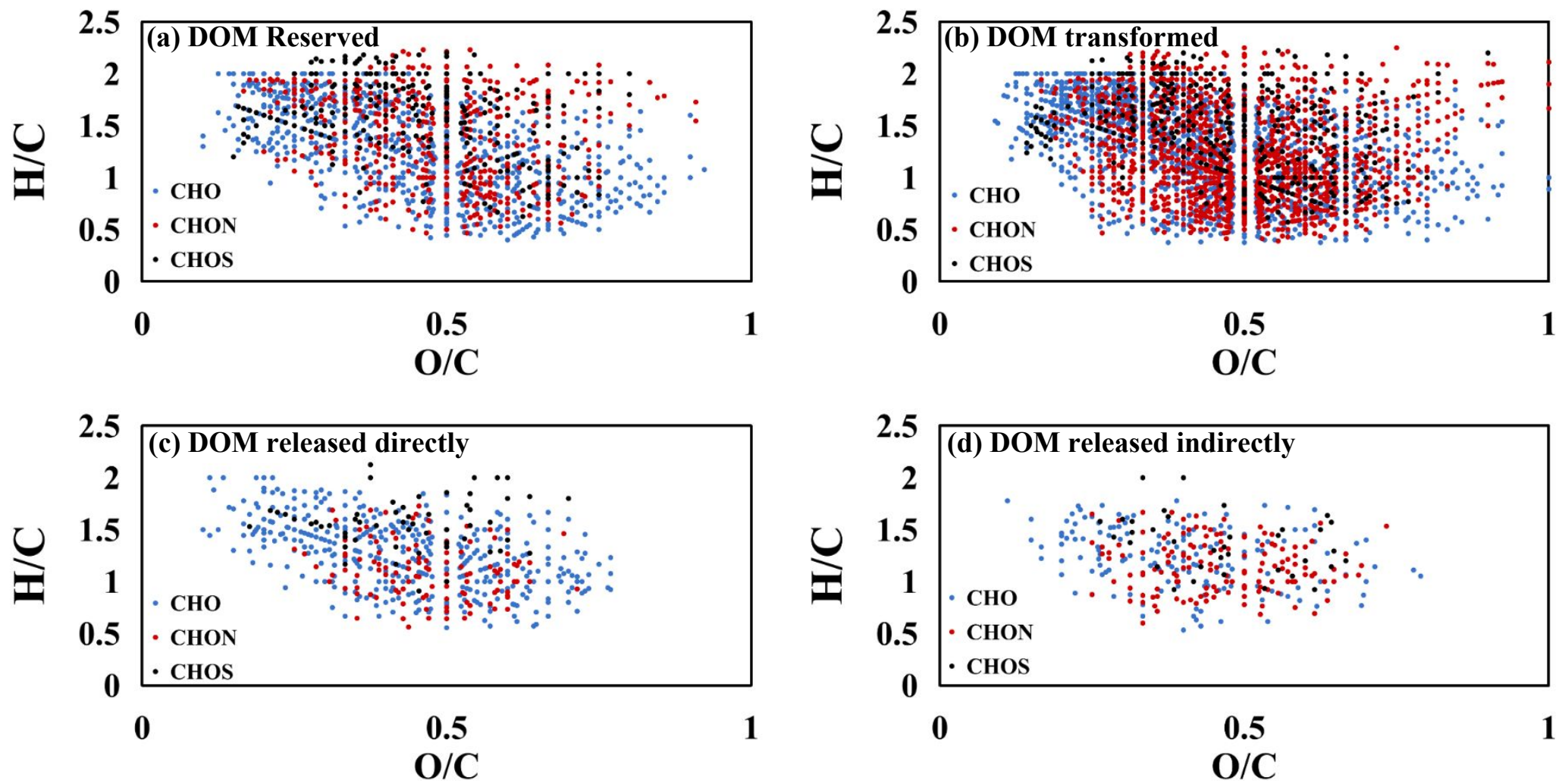

Fig. S8. Van Krevelen diagrams of different DOM pools with CHO, CHON, and CHOS compounds identified: (a) DOM reserved, (b) DOM transformed, (c) DOM released directly, (d) DOM released indirectly. 

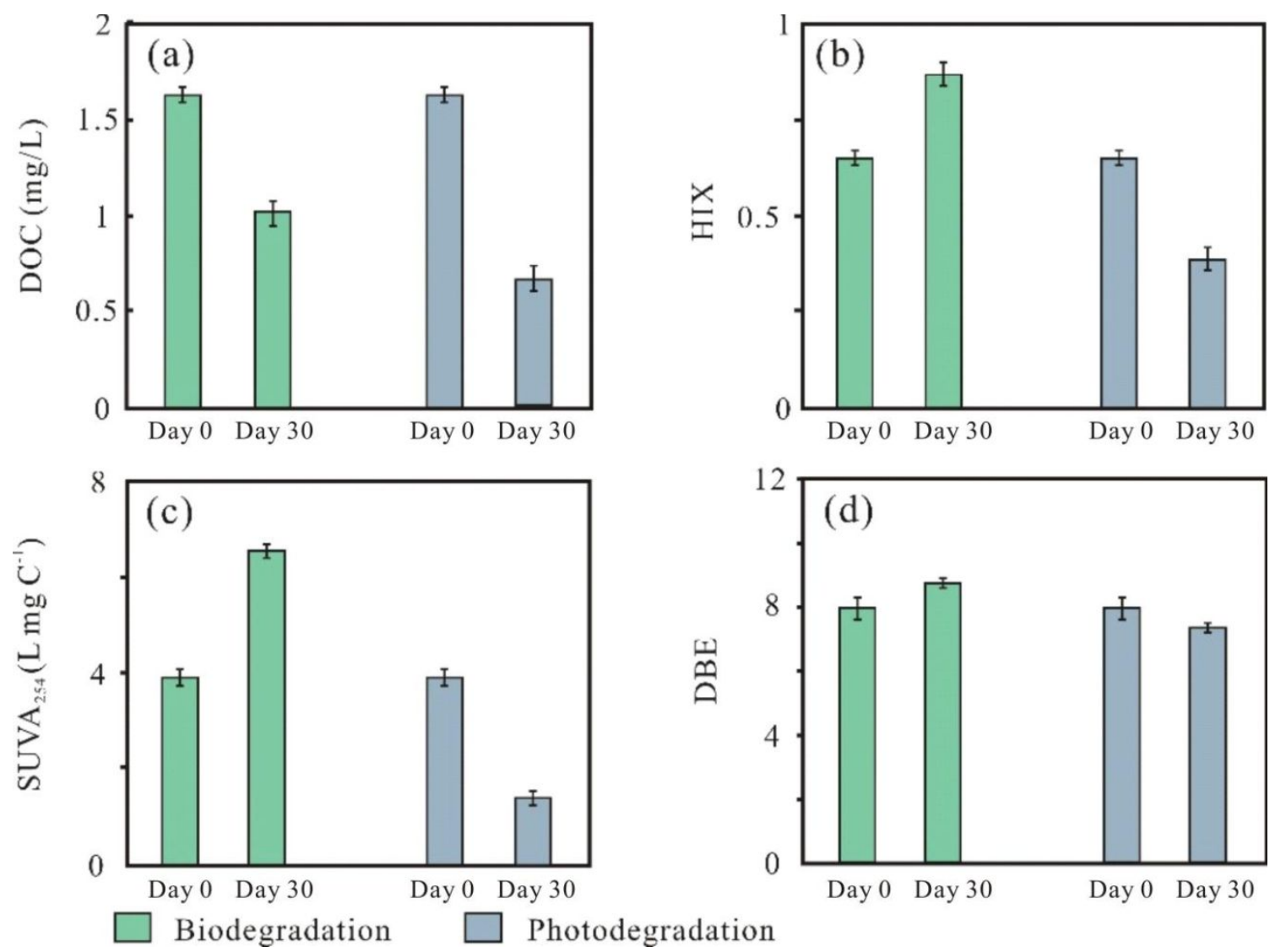

Fig. S9. Variation of DOM properties in incubation experiments: (a) DOC, (b) HIX, (c) SUVA 254 , (d) DBE. Error bars were derived from the duplicates (n=2 in biodegradation and photodegradation, respectively). 
Table S1. Intensity weighted average parameters of SRNOM identified by FT-ICR MS.

\begin{tabular}{ccccccccccccccc}
\hline Sample name & $\begin{array}{c}\text { No. of } \\
\text { formulae }\end{array}$ & CHO & CHOS & CHNO & CHONS & C & H & N & O & S & O/C & H/C & AI $I_{\text {mod }}$ & DBE \\
\hline SRNOM_1 & \multirow{2}{*}{4552} & $85.9 \%$ & $6.1 \%$ & $6.6 \%$ & $1.3 \%$ & 18.02 & 17.23 & 0.09 & 11.16 & 0.06 & 0.63 & 1.02 & 0.38 & 9.95 \\
& & $(2976)$ & $(616)$ & $(734)$ & $(226)$ & & & & & & & \\
SRNOM_2 & \multirow{2}{*}{4336} & $86.8 \%$ & $5.1 \%$ & $6.6 \%$ & $1.3 \%$ & 17.92 & 16.85 & 0.09 & 11.18 & 0.05 & 0.64 & 1.00 & 0.39 & 10.04 \\
& & $(2885)$ & $(534)$ & $(697)$ & $(220)$ & & & & & & & & \\
SRNOM_3 & 4599 & $85.5 \%$ & $6.5 \%$ & $6.6 \%$ & $1.3 \%$ & 18.07 & 17.21 & 0.09 & 11.23 & 0.07 & 0.63 & 1.01 & 0.38 & 10.01 \\
\hline
\end{tabular}


Table S2. Variation of molecular properties of S-DOM, SS-DOM, and R-DOM weighted by intensity across the water-soil interface along upstream to downstream transect.

\begin{tabular}{|c|c|c|c|c|c|c|c|c|c|}
\hline \multirow{2}{*}{ Samples } & \multicolumn{3}{|c|}{ Upstream } & \multicolumn{3}{|c|}{ Midstream } & \multicolumn{3}{|c|}{ Downstream } \\
\hline & S-DOM & SS-DOM & R-DOM & S-DOM & I SS-DOM & I R-DOM & S-DOM & SS-DOM & R-DOM \\
\hline $\mathrm{CHO}(\%)$ & 63.5 & 64.9 & 70.4 & 66.6 & 77.2 & 69.9 & 67.3 & 61.5 & 73.5 \\
\hline CHON (\%) & 5.5 & 2.9 & 10.8 & 10.9 & 7.4 & 10.4 & 15.6 & 9.6 & 11.7 \\
\hline $\mathrm{CHON}_{2}(\%)$ & 1.5 & 1,6 & 11.6 & 2.9 & 2.2 & 12.4 & 4.9 & 3.5 & 5.4 \\
\hline $\mathrm{CHON}_{3}(\%)$ & 2.1 & 2.7 & 1.8 & 2 & 1.3 & 2.1 & 2 & 3 & 2.3 \\
\hline CHOS (\%) & 27.5 & 27.6 & 4.9 & 17.6 & 11.8 & 4.9 & 9.9 & 22.1 & 5.8 \\
\hline CHNOS (\%) & 0 & 0.25 & 0.4 & 0 & 0.08 & 0.4 & 0.4 & 0.4 & 0.3 \\
\hline $\mathrm{C}$ & 17.36 & 16.83 & 20.33 & 17.79 & 16.92 & 20.38 & 17.67 & 17.62 & 20.56 \\
\hline $\mathrm{H}$ & 25.22 & 25.13 & 26 & 24.4 & 23.79 & 26.16 & 20.84 & 23.37 & 26.52 \\
\hline $\mathrm{N}$ & 0.15 & 0.14 & 0.45 & 0.23 & 0.16 & 0.47 & 0.32 & 0.26 & 0.33 \\
\hline $\mathrm{O}$ & 6.12 & 6.44 & 8.24 & 7.16 & 7.57 & 8.33 & 8.42 & 7.1 & 8.46 \\
\hline S & 0.27 & 0.28 & 0.06 & 0.18 & 0.12 & 0.06 & 0.1 & 0.22 & 0.07 \\
\hline $\mathrm{O} / \mathrm{C}$ & 0.36 & 0.39 & 0.41 & 0.41 & 0.46 & 0.41 & 0.48 & 0.46 & 0.42 \\
\hline $\mathrm{H} / \mathrm{C}$ & 1.51 & 1.56 & 1.28 & 1.43 & 1.47 & 1.28 & 1.24 & 1.39 & 1.29 \\
\hline $\mathrm{N} / \mathrm{C}$ & 0.0086 & 0.0086 & 0.0219 & 0.0127 & 0.0094 & 0.023 & 0.018 & 0.0147 & 0.0162 \\
\hline $\mathrm{S} / \mathrm{C}$ & 0.0158 & 0.0166 & 0.0027 & 0.0099 & 0.007 & 0.0027 & 0.0058 & 0.0128 & 0.0032 \\
\hline AI & 0.16 & 0.11 & 0.26 & 0.19 & 0.14 & 0.25 & 0.28 & 0.22 & 0.25 \\
\hline $\mathrm{m} / \mathrm{z}$ & 342.53 & 341.29 & 409.07 & 361.39 & 354.13 & 411.62 & 375.37 & 359.35 & 414.39 \\
\hline DBE & 5.32 & 4.84 & 7.78 & 6.2 & 5.61 & 7.76 & 7.9 & 6.56 & 7.71 \\
\hline $\operatorname{MLB}_{\mathrm{L}}(\%)$ & 65.4 & 77.5 & 18.2 & 50.6 & 65.9 & 18.6 & 32.5 & 50.4 & 19.3 \\
\hline $\begin{array}{c}\text { Black carbon } \\
(\%)\end{array}$ & 1.15 & 1.8 & 0.55 & 2.18 & 1.85 & 0.53 & 5.34 & 3.64 & 0.59 \\
\hline $\begin{array}{c}\text { Polyphenols } \\
\text { (\%) }\end{array}$ & 6.3 & 4.83 & 4.53 & 7.8 & 6.17 & 4.39 & 15.77 & 10.6 & 4.78 \\
\hline $\begin{array}{c}\text { Highly } \\
\text { unsaturated } \\
\text { compounds } \\
(\%)\end{array}$ & 27.15 & 15.84 & 80.71 & 39.34 & 26.02 & 79.72 & 46.35 & 35.31 & 78.44 \\
\hline $\begin{array}{c}\text { Unsaturated } \\
\text { aliphatics (\%) }\end{array}$ & 56.25 & 69.57 & 10.31 & 37.83 & 58.42 & 11.54 & 23.21 & 40.17 & 9.71 \\
\hline Peptides (\%) & 3.42 & 2.09 & 0.86 & 4.67 & 3.45 & 0.79 & 3.36 & 3.69 & 0.65 \\
\hline CRAM (\%) & 30.35 & 18.23 & 71.03 & 37.01 & 24.56 & 69.75 & 42.15 & 36.27 & 66.59 \\
\hline
\end{tabular}


Table S3. The list of bio-resistant, photo-resistant, bio- and photo-resistant molecules identified in incubation experiments

Note: due to the extreme long list of molecular formulae, this table is uploaded separated as a excel file. 
Table S4. Lability assessment of DOM-released directly and indirectly to rivers based on incubation experiments. The proportions were the ratio of the molecule number of DOM pool and the molecule number of initial DOM sample.

\begin{tabular}{cccc}
\hline DOM pools & Bio-resistant (\%) & Photo-resistant (\%) & Bio- and Photo-resistant (\%) \\
\hline DOM-released directly & $28.2 \pm 1.4$ & $28.4 \pm 2.1$ & $19.4 \pm 1.6$ \\
DOM-released indirectly & $18.3 \pm 1.7$ & $12.6 \pm 1.7$ & $8.9 \pm 1.4$ \\
\hline
\end{tabular}

